\title{
Emprego da vídeo-otoscopia no diagnóstico de otites externas de cães
}

\author{
Video-otoscopy use in the diagnosis of external otitis in dogs
}

\section{Cíntia Lúcia Maniscalco ${ }^{\mathrm{I}}$ James de Oliveira de Aquino ${ }^{\mathrm{II}}$ Ricardo Ferraz Britto Passos ${ }^{\mathrm{I}}$ Camila Paes Bürger ${ }^{I}$ Paola Castro Moraes $^{*}$}

\section{RESUMO}

\begin{abstract}
O objetivo deste trabalho foi estudar a utilização da vídeo-otoscopia como método diagnóstico para otites externas de cães. Para tanto, foram utilizados 20 cães adultos. O grupo A foi formado por 10 animais (cinco machos e cinco fêmeas) provenientes do canil da Universidade Estadual Paulista, Campus de Jaboticabal, sem sinais clínicos aparentes de otite externa, e o grupo $B$ foi formado por 10 animais (cinco machos e cinco fêmeas) encaminhados pelo setor de Clínica Médica do Hospital Veterinário da faculdade de Ciências Agrárias e Veterinárias, Universidade Estadual Paulista, Campus de Jaboticabal, apresentando sinais clínicos de otite. Para realização dos exames, todos os animais foram submetidos ao mesmo protocolo anestésico (acepromazina na dose de $0,1 \mathrm{mg} \mathrm{kg}^{-1}$ e tiletamina-zolazepan na dose de $10 \mathrm{mg} \mathrm{kg}^{-1}$ ). Foram diagnosticadas três otites unilaterais e 13 bilaterais, $e$ dentro dos achados estão secreção purulenta; epitélio ulcerado, hiperêmico ou hiperplásico; estenose do meato e alterações da membrana timpânica. A vídeo-otoscopia mostrou ser um método efetivo e um procedimento fundamental no auxílio diagnóstico e prognóstico das desordens auriculares.
\end{abstract}

Palavras- chave: cão, otoendoscopia, vídeo-otoscopia, otite. externa

\section{ABSTRACT}

The objective of this study was the use of videootoscopy as a method to diagnose external otitis in dogs. Hence 20 adult dogs were used. The group A was formed by 10 animals (5 males and 5 females) with no apparent symptoms of the external otitis and the group $B$, formed by 10 animals ( 5 males and 5 females) sent by the Medical Clinics section of the Veterinary Hospital at the Universidade Estadual Paulista Câmpus Jaboticabal, with otitis symptoms. All the animals tested have undergone the same anesthesic protocol (acepromazine in a dosis of $0,1 \mathrm{mg} \mathrm{kg}^{-1}$ and tiletamine-zolazepan in a dosis of $10 \mathrm{mg} \mathrm{kg}^{-1}$ ). Three unilateral and 13 bi-lateral otitis were diagnosed and the findings include purulent secretion; ulcerated, hyperemic or hyperplasic epithelium; meathus stenosis and alterations in the timpanic membrane. Video-otoscopy has shown to be an effective and a fundamental procedure to help diagnosing and prognosting auricular disorders.

Key words: video-otoscopy, otoendoscopy, otitis externa, dog.

\section{INTRODUÇÃO}

A otite externa é a mais comum das doenças do canal auditivo em cães, sendo estimado que sua prevalência está em torno de 10 a 20\%, podendo chegar a 40\% em países tropicais (LOGAS, 1994; COLE, 2004; GRIFFIN, 2006). Particularmente a otite crônica ou recorrente pode ser uma doença complicada e difícil de diagnosticar e tratar. Falhas no seu tratamento podem levar à otite externa recorrente, com ruptura da membrana timpânica, resultando em otite crônica e/ou média (SCHUNK \& AVERILL, 1983; LOGAS, 1994; ANGUS \& CAMPBELL, 2001). THIBAUT et al. (1994) observaram que $50 \%$ dos pacientes que apresentaram otite crônica resultante da forma aguda inadequadamente tratada desenvolveram quadro de otite média. ROSE (1976) constatou que a ocorrência de otite bilateral é mais comum que a unilateral.

Segundo ALÉS (2001) e ANGUS \& CAMPBELL (2001), qualquer sintoma de otite externa ou média, tais como sacudir a cabeça, prurido, secreção

'Departamento de Clínica e Cirurgia, Faculdade de Ciências Agrárias e Veterinárias, Universidade Estadual Paulista (UNESP), Campus de Jaboticabal, 14884-900, Jaboticabal, SP, Brasil. E-mail: paola.moraes@fcav.unesp.br. *Autor para correspondência. "Universidade Regional de Blumenau (FURB), Blumenau, SC, Brasil. 
no conduto auditivo externo, síndrome neurológica (ataxia, perda de equilíbrio ou outro sintoma compatível com síndrome vestibular), justificam a exploração vídeo-otoscópica.

YANAGISAWA\& CARLSON(1987)e ALÉS

(2001) definem a otoscopia como uma observação direta do conduto auditivo e da membrana timpânica. Ela é um recurso semiológico importante para a avaliação dessa membrana (ENDO \& CURI, 1998) e do meato acústico externo (EOM et al., 2000; GRIFFIN, 2006). É consenso entre diversos estudiosos sobre o assunto que a vídeo-otoscopia é método diagnóstico auxiliar para as afecções do canal auditivo, por fornecer informações importantes que condicionam o protocolo terapêutico a ser seguido, de acordo com as lesões encontradas. Por ser um procedimento não invasivo, a otoendoscopia representa também alternativa à timpanocentese para o diagnóstico da otite média (HASSEL et al.,1995).

Tendo em vista a grande importância das desordens auriculares, com este trabalho objetivou-se avaliar a utilização da vídeo-otoscopia como método diagnóstico das otites externas pela visualização direta do conduto auditivo externo e da membrana timpânica do cão.

\section{MATERIAL E MÉTODOS}

Foram utilizados 20 cães, sendo 10 provenientes do canil do Hospital Veterinário (HV) da Faculdade de Ciências Agrárias e Veterinárias (FCAV) da Universidade Estadual Paulista- UNESP, Campus de Jaboticabal, sem sinais aparentes de otite (grupo A) e os outros 10 encaminhados pelo Setor de Clínica médica do referido hospital (grupo B), com indicação para a vídeo-otoscopia por apresentarem sinais clínicos de otite. Cada grupo era formado por cinco machos e cinco fêmeas.

Para realização dos exames vídeootoscópicos, foi utilizado conjunto de vídeo-otoscopia para cães e gatos acoplado a um monitor de 14", a uma microcâmera para endoscopia e a um cabo de iluminação com fibra ótica ${ }^{a}$. Um microcomputador Pentium III e uma impressora Epson 740i foram usados para captura, armazenamento e impressão de imagens.

Os animais foram submetidos ao exame clínico e laboratorial como avaliação de rotina para escolha de protocolo anestésico adequado. Tendo em vista que todos os animais apresentaram condições ideais para serem submetidos à anestesia, utilizou-se acepromazina $^{\mathrm{b}}$, na dose de $0,1 \mathrm{mg} \mathrm{kg}^{-1}$, por via intravenosa e, após 20 minutos, tiletamina-zolazepan ${ }^{\mathrm{c}}$, na dose de $10 \mathrm{mg} \mathrm{kg}^{-1}$, por via intramuscular.
Com o animal anestesiado e em decúbito lateral, o examinador devidamente acomodado, com o monitor no campo de visão, sustentava o pavilhão auricular com uma das mãos e com a outra introduzia o otoendoscópio gradual cuidadosamente, evitando-se assim danos iatrogênicos. Inicialmente examinou-se o conduto sem limpeza para inspeção do epitélio, das secreções e para possível visualização da membrana timpânica.

Após a avaliação inicial, realizou-se limpeza, sob visão direta, de todo o conduto até a região próxima à membrana timpânica, da qual foram retiradas sujidades com curetas ou pinças do tipo jacaré. Foi feita então lavagem de todo o conduto pelo canal de trabalho do vídeo-otoscópio. Utilizou-se para isso solução fisiológica à baixa pressão para que não ocorresse ruptura da membrana timpânica. Em seguida, foi realizada outra inspeção de todas as estruturas e novas imagens foram armazenadas.

Com o término do exame, foi emitido um laudo com até seis imagens do conduto auditivo, contemplando as enfermidades quando presentes. Este foi encaminhado ao clínico responsável pelo caso, para as devidas providências quanto ao tratamento.

\section{RESULTADOS}

O protocolo anestésico utilizado foi adequado e eficaz, permitindo a realização do exame sem intercorrências. Os resultados das avaliações dos condutos auditivos dos 20 cães examinados por vídeootoscopia estão expostos na tabela 1 , salientando que um mesmo animal apresentou mais de um tipo de alteração, tanto na membrana, quanto no meato acústico externo. Vale ressaltar que seis animais do grupo A (30\%), mesmo sem sinais aparentes, apresentaram ao exame alterações de epitélio características de otite. Na avaliação das membranas timpânicas, nove animais (45\%) não apresentaram qualquer tipo de alteração, e em um animal (5\%) uma delas não pôde ser visualizada devido ao excesso de cerume. Neste, ocorreu perfuração iatrogênica durante o procedimento de lavagem e aspiração da secreção e do cerume. Deste grupo dos cães sem alteração auricular externa, foram selecionadas e armazenadas imagens dos aspectos anatômicos do meato acústico externo e da membrana timpânica normais. Dos 10 cães do grupo $\mathrm{B}$, foram selecionadas e armazenadas imagens das alterações visualizadas no meato ou na membrana timpânica (Figura 1). 
Tabela 1 - Resultados dos exames otoendoscópicos realizados nos cães - Jaboticabal-SP.

\begin{tabular}{|c|c|c|}
\hline \multicolumn{2}{|c|}{ Conclusão do laudo } & Total (\%) \\
\hline \multicolumn{2}{|c|}{ Sem alteração } & $20(n=4)$ \\
\hline \multicolumn{2}{|c|}{ Otite unilateral } & $15(n=3)$ \\
\hline \multicolumn{2}{|c|}{ Otite bilateral } & $65(n=13)$ \\
\hline \multicolumn{2}{|c|}{ Total } & $100(n=20)$ \\
\hline \multicolumn{2}{|c|}{ Aspecto analisado } & Total (\%) \\
\hline \multirow{4}{*}{ Secreção } & Leve & $5(n=1)$ \\
\hline & Moderada & $15(n=3)$ \\
\hline & Grave & $15(n=3)$ \\
\hline & Ulcerado & $5(n=1)$ \\
\hline \multirow{2}{*}{ Epitélio } & Hiperêmico & $75(n=15)$ \\
\hline & Hiperplásico & $55(n=11)$ \\
\hline \multirow{3}{*}{ Meato } & Estenose & $20(n=4)$ \\
\hline & Parasitas & $10(n=2)$ \\
\hline & $\begin{array}{l}\text { Ambas não } \\
\text { visualizadas }\end{array}$ & $15(n=3)$ \\
\hline \multirow{4}{*}{$\begin{array}{l}\text { Membrana } \\
\text { Timpânica }\end{array}$} & $\begin{array}{l}\text { Uma não } \\
\text { visualizada }\end{array}$ & $5(n=1)$ \\
\hline & Perfurada & $10(n=2)$ \\
\hline & Esbranquiçada & $20(n=4)$ \\
\hline & Abaulada & $5(n=1)$ \\
\hline
\end{tabular}

\section{DISCUSSÃO}

As vídeos-otoscopias seguiram as indicações de ALÉS (2001) e ANGUS \& CAMPBELL (2001). Os resultados das explorações do conduto auditivo e da membrana timpânica, nos grupos A e B, corroboram os resultados de YANAGISAWA \& CARLSON (1987), ENDO \& CURI (1998), EOM et al., (2000) e ALÉS (2001). Elas mostraram, com riqueza de detalhes, todas as estruturas que compõem a orelha externa como afirmaram THOMASSIN et al. (1990) e YANAGISAWA\& CARLSON (1987). Além disso, foram eficientes no diagnóstico de doenças do conduto auditivo e da membrana timpânica, confirmando os relatos de ALÉS (2001) e GRIFFIN (2006), e na excelente obtenção de fotos para documentação e prática do ensino, como indicou VENKER-van HAAGEN (1985), COLE (2004) e GRIFFIN (2006). Embora ANGUS \& CAMPBELL (2001) tenham citado a necessidade de anestesia geral inalatória, a anestesia dissociativa foi adequada, satisfatória e eficaz para a realização dos exames.

As imagens captadas do meato auditivo externo e da membrana timpânica normais do grupo A refletem as descrições, respectivamente, de ALÉS (2001), ANGUS \& CAMPBELL (2001), COLE (2004) e GRIFFIN (2006). A visualização da membrana timpânica não foi possível em alguns animais do grupo B, devido ao excesso de cerume e à presença de secreção ou estenose do meato, obstáculos já referidos por ALÉS (2001), ANGUS \& CAMPBELL (2001) e MURPHY (2001). Quando havia secreção ou cerume em excesso, realizou-se lavagem com solução fisiológica, como fizeram ANGUS \& CAMPBELL (2001), embora em um dos animais do grupo A, durante esse processo, tenha ocorrido perfuração iatrogênica de uma das membranas timpânicas, fato já alertado por ALÉS (2001). A lavagem, segundo ANGUS \& CAMPBELL (2001), reforça a importância da vídeo-otoscopia como método não somente diagnóstico, mas também auxiliar na terapia da otite externa. Entretanto, tanto a limpeza, como a lavagem, feitas sem o auxílio desse equipamento, podem aumentar os riscos de perfuração iatrogênica da membrana timpânica (ALÉS, 2001).

Os achados da vídeo-otoscopia coincidem com aqueles relatados na literatura. No conduto auditivo, foram observados os mesmos achados de ALÉS (2001): hiperemia, hiperplasia, estenose e ulceração do epitélio; parasitas e pelos. Na membrana timpânica, foram observados os mesmos achados de ENDO \& CURI (1998) eANGUS \& CAMPBELL(2001), como alterações de coloração, perfuração, hiperemia e abaulamento. A ocorrência de abaulamento, segundo HOSKINS (1997), é indicativa de acúmulo de sangue, pus ou fluido na orelha média, o que não foi possível comprovar, pois a membrana estava intacta.

Dos 20 animais examinados, 13 (65\%) tinham otite externa, sendo três (15\%) unilaterais, o que confirma a afirmação de ROSE (1976), quando relata a prevalência da bilateralidade da afecção. As imagens dos achados foram selecionadas e armazenadas em computador para emissão de laudos, prática de ensino, documentação médica, além de servirem como argumento para explicação ao cliente da situação do paciente, seguindo as indicações de YANAGISAWA \& CARLSON (1987); ALÉS (2001); ANGUS \& CAMPBELL (2001) e MURPHY (2001).

\section{CONCLUSÕES}

A vídeo-otoscopia mostrou ser método efetivo para visualização rápida e segura do conduto auditivo externo e da membrana timpânica. É um procedimento útil no auxílio diagnóstico e prognóstico das desordens auriculares. Acoplado ao computador e à impressora, o otoendoscópio possibilita não só mostrar ao cliente, como também armazenar e documentar os achados patológicos ou não. Esses recursos permitem a emissão de laudos, a cada exame, para servir como argumento de explicação da situação do paciente em relação ao problema. 


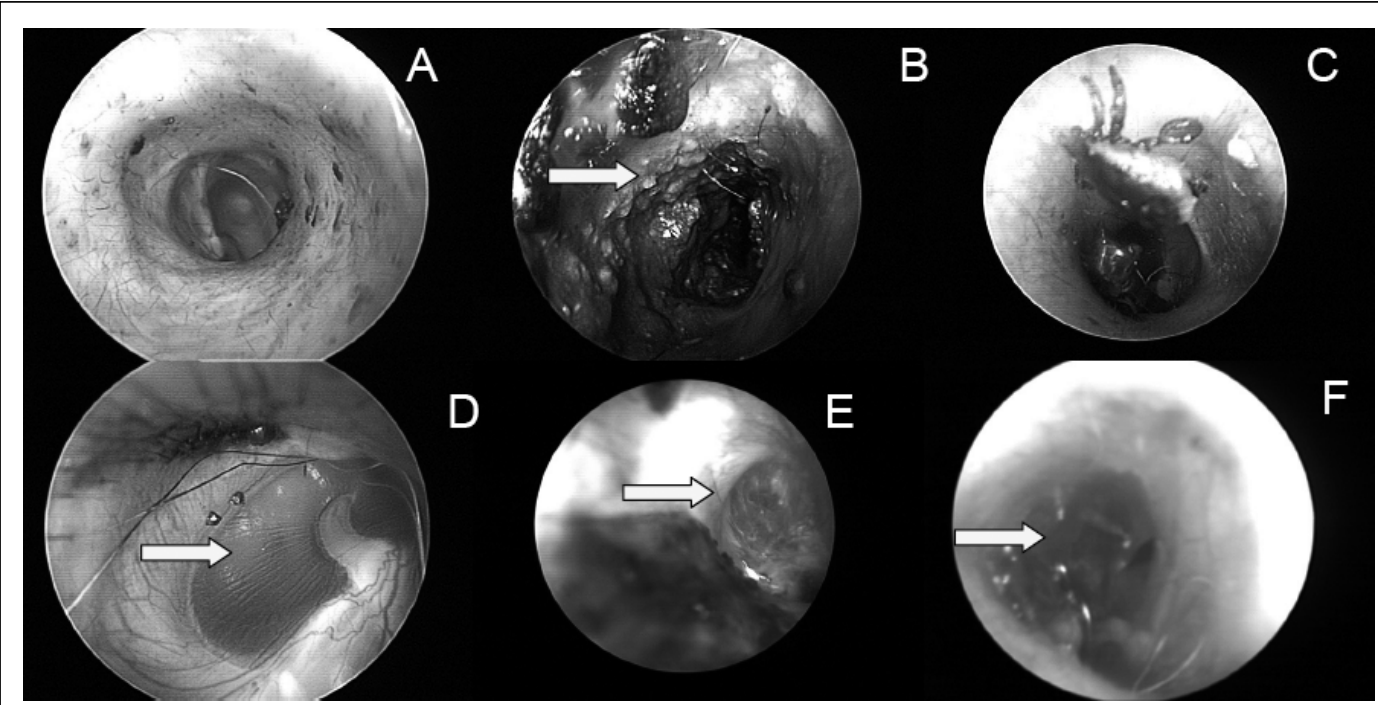

Figura 1 - Imagem otoendoscópica de cão. A) meato acústico externo normal. Pequena quantidade de cerume e pelos. B) excesso de cerume, hiperplasia de epitélio (seta). C) presença de parasito (carrapato). D) membrana timpânica normal (seta). E) membrana timpânica esbranquiçada (seta). F) membrana timpânica perfurada (seta).

\section{AGRADECIMENTOS}

Os autores agradecem à Faculdade de Ciências Agrárias e Veterinárias, Campus de Jaboticabal, e à Coordenação de Aperfeiçoamento de Pessoal de Nível Superior (CAPEs), pela bolsa de estudo concedida ao Mestre James de Oliveira de Aquino. Este estudo foi financiado pela Fundação de Amparo à Pesquisa do Estado de São Paulo (FAPESP).

\section{FONTES DE AQUISIÇÃO}

a- KARL STORZ GMBII \& CO

b- ACEPRAN 1\% - Laboratório Univet

c- ZOLETIL - Laboratório Virbac

\section{COMITÊ DE ÉTICA E BIOSSEGURANÇA}

O presente estudo foi avaliado e aprovado pela Comissão de Ética e Bem-estar Animal (CEBEA) da Faculdade de Ciências Agrárias e Veterinárias- UNESP, Campus de Jaboticabal (Protocolo 9902).

\section{REFERÊNCIAS}

ALÉS, J.F.A. Video-otoscopia en el perro. Disponível em: http://www.aevedi.org/convirtual.htm. Acesso em 23 jul. 2001.

ANGUS, J.C.; CAMPBELL, K.L. Uses and indications for Video-otoscopy in small animal practice. Veterinary Clinics of North America: Small Animal Practice, v.31, n.4, p.809828, 2001.

COLE, L.K. Otoscopy evaluation of the ear canal. Veterinary Clinics of North America: Small Animal Practice, v.34, n.2, p.397-410, 2004.

ENDO, L.H.; CURI, S.B. Otoscopia e timpanometria no diagnóstico da otite média secretora. Jornal de Pediatria, v.74, n.5, p.353-354, 1998.
EOM, K.D. et al. Canalographic evaluation of the external ear canal in dogs. Veterinary Radiology Ultrasound, v.41, n.2, p.231-234, 2000 .

GRIFFIN, C.E. Otitis techniques to improve practice. Clinical Techniques in Small Animal Practice, v.21, n.3, p.96105, 2006 .

HASSEL, D.M. et al. Endoscopy of the auditory tube diverticula in four horses with otitis media/interna. Journal American Veterinary Medical Association, v.207, n.8, p.1081-1084, 1995.

HOSKINS, J.B. Pediatria veterinária. 2.ed. Rio de Janeiro: Interlivros, 1997. 601p.

LOGAS, D.B. Diseases oh the ear canal. Veterinary Clinics of North America: Small Animal Practice, v.24, n.5, p.905909, 1994

MURPHY, K.M. A review of techniques for the investigation of otites externa and otites media. Clinical Techniques in Small Practice, v.16, n.3, p.236-241, 2001.

ROSE, W.R. Otitis externa - I: incidence. Veterinary Medical/ Small Animal Clinics, n.5, p.638-640, 1976.

SCHUNK, K.L.; AVERILL, D.R. Jr. Peripheral vestibular syndrome in the dogs: a review of 83 cases. Journal of the American Veterinary Medical Association, v.182, n.12, p.1354-1357, 1983.

THIBAUT, M.V. et al. Contribuición al estudio de la otitis externa del perro. Archivos de Medicina Veterinária., v.26, n.2, p.85-95, 1994.

THOMASSIN, J.M. et al. Otoendoscopy: applications in the meiddle ear surgery. Revue de Laringologie Otologie Rhinologie, v.111, n.5, p.475-477, 1990.

VENKER-van HAAGEN, A.J. Otoscopy, rhinoscopy, and bronchoscopy in small animal clinics. Veterinary Quarterly, v.7, n.3, p.222-224, 1985.

YANAGISAWA, E.; CARLSON, R.D. Telescopic videootoscopy using a compact home video color camera. Laringoscope, v.97, n.11, p.1350-1355, 1987. 